\title{
Circulating tumor cell methylation profiles reveal the classification and evolution of non-small cell lung cancer
}

\author{
Jia-Hao Jiang ${ }^{1 \#}$, Jian Gao ${ }^{1 \#}$, Chang-Yue Chen ${ }^{2 \#}$, Yong-Qiang Ao ${ }^{1}$, Jing Li $^{2}$, Yuan $\mathrm{Lu}^{2}$, Wang Fang ${ }^{3}$, \\ Hai-Kun Wang ${ }^{4}$, Douglas Guedes de Castro ${ }^{5}$, Mariacarmela Santarpia ${ }^{6}$, Masaki Hashimoto ${ }^{7}$, \\ Yun-Feng Yuan ${ }^{1}$, Jian-Yong Ding ${ }^{1}$
}

${ }^{1}$ Department of Thoracic Surgery, Zhongshan Hospital, Fudan University, Shanghai, China; ${ }^{2}$ Research and Development Department, Shanghai Zhiyi Biomedical Technology Company, Shanghai, China; ${ }^{3}$ Academic Marketing Department, Jilin Province JinKangAn Pharmaceutical Company, Dunhua, China; ${ }^{4}$ CAS Key Laboratory of Molecular Virology and Immunology, Pasteur Institute of Shanghai, Chinese Academy of Sciences, Shanghai, China; ${ }^{5}$ Department of Radiation Oncology, AC Camargo Cancer Center, São Paulo, Brazil; ${ }^{6}$ Medical Oncology Unit, Department of Human Pathology “G. Barresi”, University of Messina, Messina, Italy; ${ }^{7}$ Department of Thoracic Surgery, Hyogo College of Medicine, Nishinomiya, Japan

Contributions: (I) Conception and design: JH Jiang, J Gao, CY Chen, YF Yuan, JY Ding; (II) Administrative support: HK Wang, DG de Castro, M Santarpia; (III) Provision of study materials or patients: JH Jiang, J Gao, YF Yuan, JY Ding; (IV) Collection and assembly of data: CY Chen, YQ Ao, J Li, Y Lu, W Fang; (V) Data analysis and interpretation: JH Jiang, CY Chen, J Li, Y Lu, W Fang, YQ Ao; (VI) Manuscript writing: All authors; (VII) Final approval of manuscript: All authors.

"These authors contributed equally to this work.

Correspondence to: Jian-Yong Ding; Yun-Feng Yuan. Department of Thoracic Surgery, Zhongshan Hospital, Fudan University, 180 Fenglin Road, Shanghai 200032, China. Email: ding.jianyong@zs-hospital.sh.cn; yuan.yunfeng@zs-hospital.sh.cn.

Background: The ability of circulating tumor cells (CTCs) to identify lung adenocarcinoma (LUAD) and lung squamous cell carcinoma (LUSC) could improve pathological diagnosis and the selection of treatments for non-small cell lung cancer (NSCLC). Previous studies have shown that deoxyribonucleic acid (DNA) methylation exhibits cell and tissue specificity. Thus, we aimed to explore the methylation status of CTCs in LUAD and LUSC and identify the potential biomarkers.

Methods: We first analyzed Infinium 450K methylation profiles obtained from The Cancer Genome Atlas and Gene Expression Omnibus. We then performed whole-genome sequencing of CTCs in tumor and matched normal lung tissues and white blood cells from 6 NSCLC patients.

Results: The bioinformatics analysis revealed a NSCLC-specific DNA methylation marker panel, which could accurately distinguish between LUAD and LUSC with high diagnostic accuracy. The whole-genome sequencing of CTCs in NSCLC patients also showed 100\% accuracy for distinguishing between LUAD and LUSC based on the CTC methylation profiles. To investigate the function of CTCs, we further analyzed similar and different methylation profiles between the CTCs and their primary tumors, and found very high similarities between the CTCs and their primary tumor tissues, indicating that these cells inherit information from primary tumors. However, the CTCs also displayed some characteristics that differed to those of primary tumor tissues, which suggest that CTCs acquire some unique characteristics after migrating from the primary tumor; these characteristics may partly explain the ability of tumor cells to evade immune surveillance.

Conclusions: Our findings provide insights into the potential use of CTCs in the pathological classification of NSCLC patients. Our findings also show how CTC primary tumor inheritance and CTC evolution affect metastasis and immune escape.

Keywords: Non-small cell lung cancer (NSCLC); circulating tumor cells (CTCs); DNA methylation

Submitted Nov 23, 2021. Accepted for publication Feb 16, 2022.

doi: $10.21037 /$ tlcr-22-50

View this article at: https://dx.doi.org/10.21037/tlcr-22-50 


\section{Introduction}

Lung cancer is one of the most common and deadliest forms of cancer worldwide (1). Approximately $85 \%$ of lung cancers are non-small cell lung cancers (NSCLCs), which include lung adenocarcinoma (LUAD) and lung squamous cell carcinoma (LUSC). As the main treatment options are determined according to the histologic features and the molecular profile, pathological diagnosis is key in NSCLC treatment. Currently, pathological diagnoses are based on the morphologic features or on immune-cytochemical and immune-histochemical analyses of NSCLC tissues, or cytologic samples obtained from a surgical biopsy, bronchoscopy, or bronchial brushing (2), and it is difficult to make a pathological diagnosis in cases in which tumor biopsy or cytology material is not adequate.

Circulating tumor cells (CTCs) are cancer cells that disseminate from primary or metastatic sites into the peripheral blood. CTCs have great potential as diagnostic and prognostic biomarkers, and could be used to guide the individualized treatment of lung cancer patients. In the lung cancer diagnostic field, the CTC detection rate is approximately $87 \%$ among those who have $>3$ CTCs per $3.2 \mathrm{~mL}$ of blood (3). Further, CTCs carry information about the primary tumor cells and could be considered an alternative means of tumor subtype classification (4).

In metastasis, the methylation status of the primary tumor is inherited by the metastatic tumor. Previous studies have shown that deoxyribonucleic acid (DNA) methylation exhibits cell and tissue specificity (5), and changes in DNA methylation play an important regulatory role in the development of cancer $(6,7)$. Indeed, both genome-wide hypomethylation and hypermethylation modifications have the ability to alter the expression of neighboring genes and contribute to cancer phenotypes $(7,8)$. DNA methylation has been extensively investigated in primary tumors (9); however, the events that shape the DNA methylome during metastatic dissemination are largely uncharacterized $(10,11)$. Overall, knowledge of the methylation profile of CTC DNA may broaden our understanding of tumor cell origin and evolution.

In this study, we combined immunostaining fluorescence in situ hybridization (FISH)-based CTC identification, laser capture microdissection-based CTC capture, and single-cell resolution DNA methylation to explore CTC methylation signatures in the origin, classification, and evolution of these cells in NSCLC. Our study provides a genome-wide DNA methylation landscape of primary tumor tissues,
CTCs, matched normal lung tissues, and white blood cells (WBCs) in 6 NSCLC patients. The results demonstrate that CTCs can be used as an effective blood-based method for the classification of LUAD and LUSC. The results also showed that both CTC primary tumor inheritance and CTC evolution affect metastasis and immune escape. We present the following article in accordance with the MDAR reporting checklist (available at https://tlcr.amegroups.com/ article/view/10.21037/tlcr-22-50/rc).

\section{Methods}

\section{Characteristics of patients and samples}

The clinical characteristics and molecular profiles, including methylation data for a training cohort of 553 tumor samples and 60 matched adjacent normal tissue samples, and a validation cohort of 288 tumor and 14 matched normal samples, were obtained from The Cancer Genome Atlas (TCGA). A separate validation cohort of 37 tumor samples and 74 normal samples was obtained from Gene Expression Omnibus (GEO). Another separate validation cohort of 6 tumor and 6 matched normal samples was obtained from Zhongshan Hospital of Fudan University, Shanghai, China. Matched adjacent normal tissue samples and WBCs were collected at the same time as the tumor tissue from each patient, and subjected to a histological analysis to confirm that there was no evidence of cancer. The clinical characteristics of all the patients are summarized in Table S1. None of the 6 patients received any additional treatment apart from the surgery. Written informed consent was obtained from the patients, and ethical approval was obtained from the Zhongshan Hospital Research Ethics Committee (No. Y2019-187). All procedures performed in this study involving human participants were in accordance with the Declaration of Helsinki (as revised in 2013).

\section{Experimental method details}

\section{Subtraction enrichment of CTCs and identification of aneuploid CTCs}

The enrichment and identification of CTCs were performed in accordance with the instructions of the CTCseq ${ }^{\mathrm{TM}} \mathrm{kit}$ (Majorbio). The samples were fixed on slides, and then counted and photographed. Each suspicious tumor cell coordinate was recorded to facilitate subsequent target cell identification. The identification principle of CTCs is that they are (I) negative for cluster of differentiation (CD)45 
and (II) positive for chromosome 8 heteroploidy. The slides were stored at $-20^{\circ} \mathrm{C}$.

\section{Laser capture microdissection}

The samples were loaded onto the stage of a Zeiss PALM MicroBeam (Zeiss) under a $\times 40$ objective. After microdissection with a 355-nm laser beam, the target cells were collected on an AdhesiveCap 200 opaque cover (Zeiss). The $10-\mu \mathrm{L}$ reaction volume contained $5 \mu \mathrm{L}$ of M-Digestion Buffer (2×), $0.5 \mu \mathrm{L}$ of proteinase K (EZ DNA Methylation-Direct $\left.{ }^{\mathrm{TM}} \mathrm{Kit}, \mathrm{Zymo}\right)$, and $4.5 \mu \mathrm{L}$ of nucleasefree water (Ambion). The reagents were mixed and placed on the AdhesiveCap 200 opaque cover; the tube was briefly microcentrifuged, and the reaction was incubated in a thermal cycler for $20 \mathrm{~min}$ at $50{ }^{\circ} \mathrm{C}$, with a $4{ }^{\circ} \mathrm{C}$ hold. The samples were stored at $-20^{\circ} \mathrm{C}$.

\section{WGBS analysis}

\section{Tumor DNA extraction}

Genomic DNA extraction from freshly frozen normal or cancer tissues or WBCs was performed with a QIAamp DNA Mini Kit (Qiagen) in accordance with the manufacturer's recommendations. DNA was extracted from approximately $0.5 \mathrm{mg}$ of tissue and stored at $-20{ }^{\circ} \mathrm{C}$; the samples were analyzed within 1 week of preparation.

\section{Bisulfite conversion of genomic DNA and WGBS}

Bisulfite conversion was performed using the EZ DNA Methylation-Lightning ${ }^{\mathrm{TM}}$ Kit (Zymo Research). Wholegenome bisulfite sequencing (WGBS) was performed using the KAPA Hyper Prep Kit (Roche) with several modifications, as previously described (12). The WGBS libraries of tissue and WBCs were sequenced with pairedend flow cell lanes in the HiSeq4000 system (Illumina) for 150 cycles.

\section{Capture and sequencing}

Capture was performed using the SeqCap Epi CpGiant Enrichment Kit (Roche) in accordance with the manufacturer's instructions. Briefly, 4-6 bisulfite-treated libraries (200 ng/sample) were hybridized to the SeqCap Epi probe pool; the beads were captured, washed, amplified, quantified, and qualified as directed in the protocol. The captured pooled library (tissue $2 \mathrm{~N}$ ) was sequenced using the Illumina HiSeq $\mathrm{X}$ Ten system with a 150 -bp paired-end model.

\section{Bisulfite conversion and single-cell whole-genome bisulfite library preparation}

The library was produced according to a previously published protocol (12). In brief, after cell lysis for $20 \mathrm{~min}$, the CTC samples were subjected to bisulfite conversion using the EZ DNA Methylation-Direct ${ }^{\mathrm{TM}}$ Kit (Zymo) in accordance with the manufacturer's instructions. The bisulfite-converted DNA was then synthesized using Klenow exo-(Enzymatics) with a truncated Illumina P5 adapter (5'-CTACACGACGCT CTT CC GATCTNNNNNN-3') followed by a random hexamer at the 3 'end. This step was repeated 4 additional times for preamplification. The excess primers were removed using exonuclease I (New England Biolabs). Following purification, the 2 nd strands were synthesized similarly but using a truncated P7 Illumina adapter (5'-AGACGTGTGCTCTTCCGATCTNNNN NN-3'). The final library was amplified using the KAPA HiFi HotStart ReadyMix (Kapabiosystems) with NEB primers (universal primer and index primer). The amplified libraries were purified twice with $0.9 \times$ AMPure XP beads (Beckman Coulter), and quantified using Qubit ds HS dye and a 2100 Bioanalyzer (Agilent Technologies). The final quality-ensured libraries were sequenced with a HiSeq4000 system (Illumina) for 150 cycles.

\section{Quantification and statistical analysis}

\section{Processing methylation microarray data}

The DNA methylation data were obtained from TCGA analysis of 485,000 sites generated using Infinium 450K Methylation Array, and the following GSE datasets: GSE85845, GSE83842, GSE66087, GSE63704, and GSE53051. The microarray data (level 3 in TCGA and processed matrix files in the GEO database) provided the methylation levels of the individual CpG sites. The methylation levels for the two cancer subtypes (i.e., LUAD and LUSC) and normal lung tissues were extracted. Six matched cohorts (cancer, normal, bulk WBCs, and CTCs per patient) were obtained by WGBS and analyzed as described.

\section{Building the multiclass classifier}

For each of the three subtypes of LUAD and LUSC cancer and corresponding normal tissue samples from TCGA, we randomly split the full TCGA 450K data set into training and validation sets at a 2:1 ratio. We first performed prescreening to remove excessive noise from 
the training data using the Dunn test. First, a CpG site methylation level was marked as "not available" (NA) if methylation measurements were not available for more than half of the CpG sites. Second, any samples that had missing methylation levels for more than $5 \mathrm{k} \mathrm{CpG}$ sites were marked as "NA". Third, for each set of comparisons, 1 type of sample was compared against the other two types of samples. A list of markers with significant methylation differences $\geq 0.2$ and $\mathrm{P}$ values $<0.05$ between LUAD and LUSC, and significant methylation differences $\geq 0.1$ and $P$ values $<0.05$ between LUAD and normal tissues or LUSC and normal tissues were retained for future analysis. The Benjamini-Hochberg procedure was used to control the false discovery rate at a significance level of 0.05 . For the multinomial classification, we used logistic regression with the L2 regularization model (Ridge), and the tuning parameter was determined by the expected generalization error estimated from the 5 -fold cross-validation. A multiclass prediction system was constructed to predict a cancer subtype or normal sample in the validation data using the selected features. A confusion matrix and receiver operating characteristic curves were also produced to evaluate sensitivity and specificity in addition to prediction accuracy.

All the data analyses were conducted by custom-made bash and $\mathrm{R}$ and Python scripts ( $\mathrm{R}$ version $=3.4 .2$, Python version $=3.7 .2$ ) with the dunn.test $(\mathrm{R})$ and sklearn (Python) packages.

\section{WGBS processing}

After tissue and CTC WGBS sequencing, an initial quality assessment of the data was performed using FastQC (https://www.bioinformatics.babraham.ac.uk/projects/ fastqc). Adaptor sequences, low-quality ends, and $6 \mathrm{bp}$ from both the $5^{\prime}$ and $3^{\prime}$ ends of reads were removed with Trim Galore (v0.4.2, http://www.bioinformatics.babraham. ac.uk/projects/trim_galore/; parameters: --clip_R1 6 --clip_ R2 6 --three_prime_clip_R1 6 --three_prime_clip_R2 6). Trimmed reads were aligned to the hg19/GRCh37 human genome using Bismark with the alignment tool Bowtie2 (v2.2.9) (main parameter: --score-min L, 0, 0.2) $(13,14)$. Finally, methylation cells were extracted after deduplication using Bismark. Only CpG sites with a depth of coverage $\geq 3 \times$ were considered for the methylation analysis.

\section{Differential methylation analysis and enrichment analysis \\ Differentially methylated CpGs were assessed using a}

methylKit (R package) (15). Under the sliding linear model (SLIM) method, a $\mathrm{P}$ value $<0.05$ indicated differential methylenation (16). We selected differentially methylated CpG sites (DMCs) detected in 2 of the 6 patients for the future functional pathway analysis. Transcription factor binding sites (TFBSs) in DMCs were calculated using i-cisTarget (https://gbiomed.kuleuven.be/apps/lcb/ i-cisTarget/) with a full motif analysis (17). The Kyoto Encyclopedia of Genes and Genomes (KEGG) analysis with a hypergeometric test implemented in the clusterProfiler $\mathrm{R} /$ bioconductor package was performed using ClueGO (Vocci and London, 1997). The annotation used for the CpGIslands and RefSeq genes was performed using the genomation toolkit in the $\mathrm{R} /$ bioconductor package.

\section{KEGG pathway enrichment analysis based on genomic} features

Individual CpGs were mapped to genes and their promoters using the RefSeq gene annotation from the University of California Santa Cruz genome browser (https://genome. ucsc.edu; date: 10/10/2020). Promoters were defined as the $\pm 2 \mathrm{~kb}$ region around the transcription start site. Mapping to superenhancer regions was based on dbSUPER (http://asntech.org/dbsuper/), an integrated database of superenhancers that provides a list of genes associated with each region. Each genomic feature was interrogated for differential methylation in the same manner as that for genomic tiles. Similar genes corresponding to genomic features with a normal $\mathrm{P}$ value $>0.05$ and a methylation difference $<10 \%$ were considered for the enrichment analysis between CTCs and matched tumor tissues. Differential genes corresponding to genomic features with a normal $\mathrm{P}$ value $<0.05$ and an absolute methylation difference $>0.1$ were considered for the enrichment analysis between CTCs and matched tumor tissues. The gene set enrichment analysis was performed using a hypergeometric test implemented in the clusterProfiler R/bioconductor package. Gene sets with an adjusted $\mathrm{P}$ value $<0.05$ were considered statistically significant.

\section{Results}

\section{Identification and validation of cancer-specific differential methylation $C p G$ sites}

To explore NSCLC-specific DNA methylation markers, we first analyzed Infinium $450 \mathrm{~K}$ methylation profiles obtained from TCGA (see Figure 1). We hypothesized that 


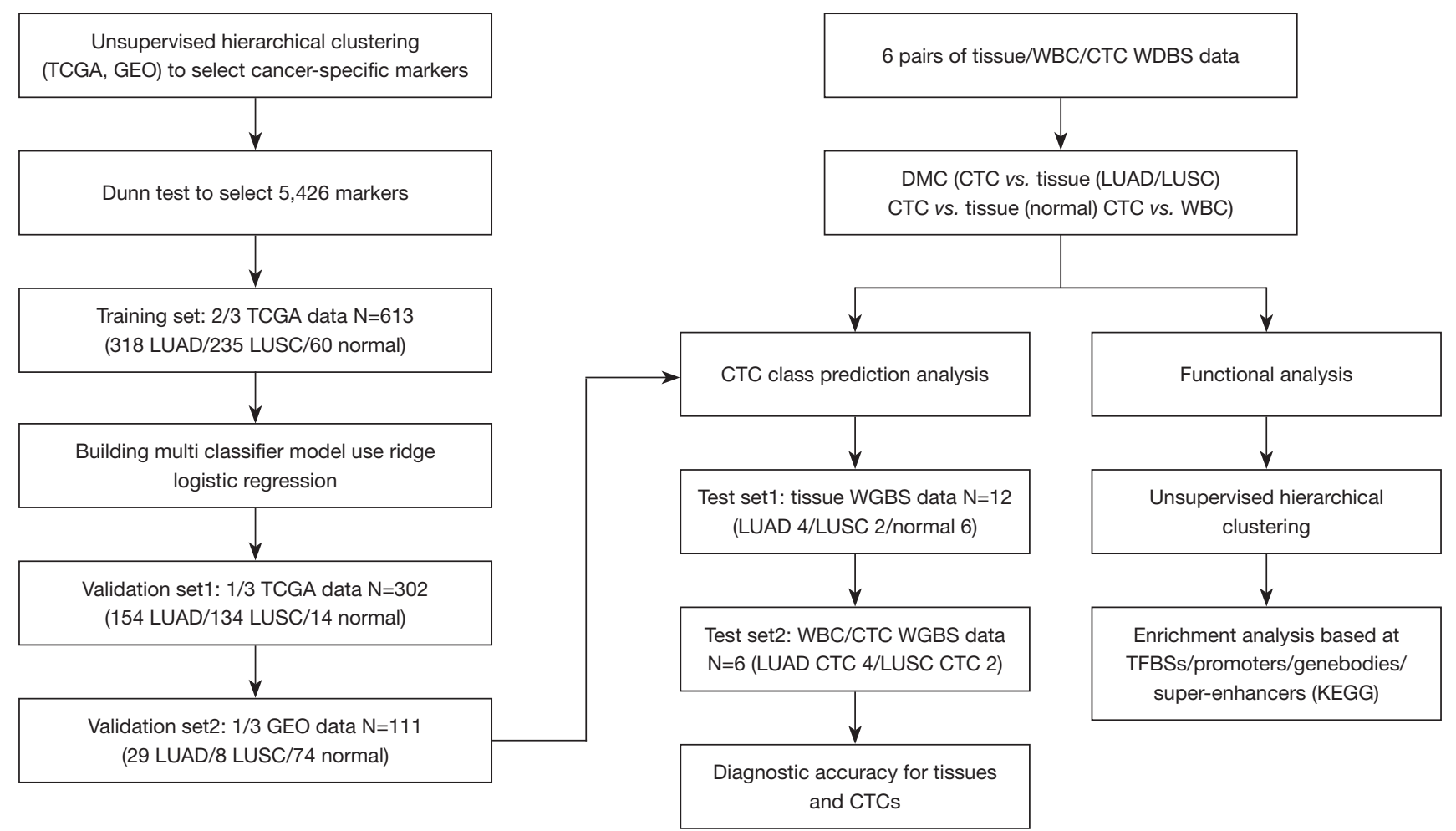

Figure 1 Workflow chart of data generation and analysis. TCGA methylation data was used to cluster and identify 5,426 features. A multiclassification model was built based on the selected features. 2/3 of the TCGA data were used for training, and 1/3 of the TCGA and the GEO data were used for validation. Patient-WGBS data were used for prediction model testing and the functional analysis. WBC, white blood cell; TCGA, The Cancer Genome Atlas; GEO, Gene Expression Omnibus; WGBS, whole-genome bisulfite sequencing; DMCs, differentially methylated CpG sites; LUAD, lung adenocarcinoma; LUSC, lung squamous cell carcinoma; Normal, normal tissues; CTC, circulating tumor cell; KEGG, Kyoto Encyclopedia of Genes and Genomes.

the most appropriate methylation differences (LUSC $v s$. LUAD vs. normal) would lead to the best performance in both clustering and classification. Thus, we started with 5 cutoff parameters of beta differences (greater than the cutoff) of $0.10,0.15,0.20,0.25$, and 0.30 among LUAD versus LUSC versus normal. The same cutoff parameters were used for LUAD and LUSC compared to normal controls. Four groups of features with each fixed parameter set were identified according to whether the difference in methylation met the cutoff for: (I) LUAD_LUSC_Normal specific; (II) LUAD_specific; (III) LUSC_specific; and (IV) Normal_specific. We assessed 4 combinations of specific probes [(I); (I) + (II) + (III), (I) + (II) + (III) + (IV), and (I) + (IV)] under each fixed parameter set, resulting in $5 \times 5 \times 4=100$ mixed samples. Finally, we obtained the optimal parameters for LUAD_LUSC_diff $\geq 0.20$, LUAD_Normal_diff $\geq 0.10$, and LUSC_Normal_diff $>0.10(\mathrm{P}<0.05)$. The clustering results are shown in Figure $2 \mathrm{~A}$.
Under the optimal parameters, we detected 5,426 DMCs, including 5,426 LUAD-LUSC cancer-specific DMCs, 1,409 LUAD-normal specific DMCs, and 2,919 LUSC-normal specific DMCs (see https://cdn.amegroups.cn/static/public/ tlcr-22-50-1.xlsx). Based on the differential methylation of the CpG sites, we were able to distinguish LUAD, LUSC, and normal tissues with diagnostic accuracies of $97.5 \%$, $95.7 \%$, and $100 \%$, respectively (see Figure $2 B$ and Table S2).

To assess the diagnostic accuracy of the methylation marker panel, we then applied the methylation panel to $1 / 3$ of TCGA validation cohort 1 and GEO validation cohort 2 (see Figure 2C,2D). The diagnostic accuracy of the panel for LUAD, LUSC, and normal tissues was $98.1 \%, 94.8 \%$, and $100 \%$, respectively, in $1 / 3$ of TCGA validation cohort 1 (see Figure $2 C$ and Table S3), and $86.2 \%, 87.5 \%$, and $98.6 \%$ in GEO validation cohort 2 (see Figure $2 D$ and Table S4), respectively. These results demonstrate the robust nature of the methylation panel in identifying the presence of 
A

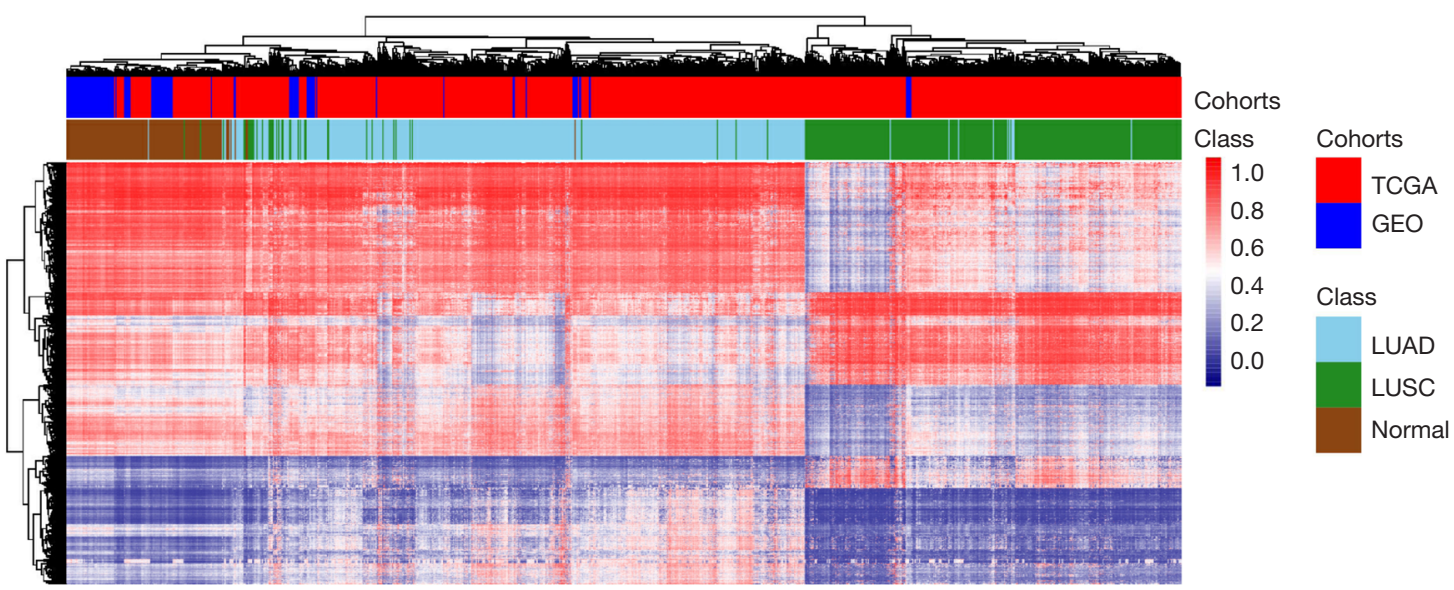

B

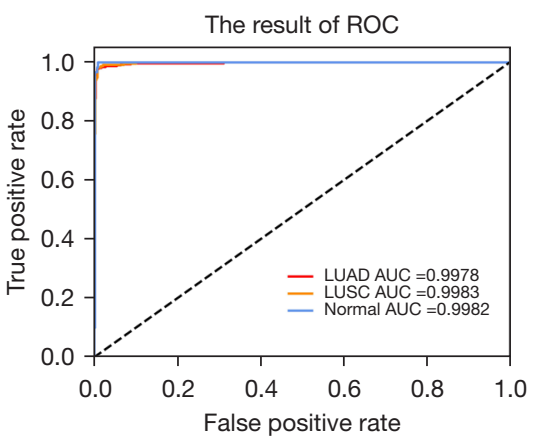

C

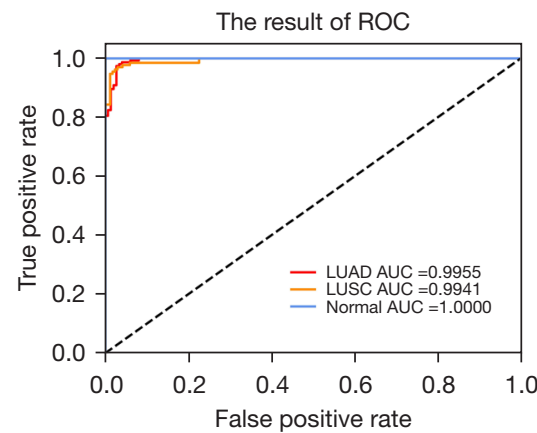

D

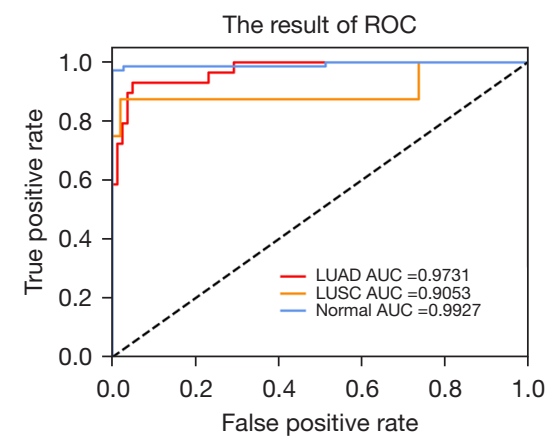

Figure 2 Clustering and receiver operating characteristic analyses of the discovery and validation sets using the 5,426 CpG markers identified in TCGA cohort. (A) DNA methylation signatures can identify LUAD, LUSC, and NORMAL in TCGA and GEO cohorts. Shown are the unsupervised hierarchical clustering and heat maps associated with the methylation profile of $501 \mathrm{LUAD}$ samples (skyblue), 377 LUSC samples (green), and 148 normal samples (brown) in TCGA (red) and GEO (blue) cohorts with a panel of 5,426 CpG markers. Each column represents an individual patient, and each row represents an individual CpG marker. The color scale shows the DNA methylation level. (B) ROC curve of the diagnostic prediction model with methylation markers in 2/3 of the TCGA training cohort. (C) $1 / 3$ of the TCGA validation cohort 1; (D) GEO validation cohort 2. LUAD, lung adenocarcinoma; LUSC, lung squamous cell carcinoma; TCGA, The Cancer Genome Atlas; GEO, Gene Expression Omnibus.

malignancy and its NSCLC subtype classification.

\section{Cancer-specific methylation signature validation in tissues and CTCs}

To validate the clinical value of the methylation panel, we next performed the WGBS analysis of the tumor tissue samples, matched normal lung tissue samples, CTCs, and WBCs from 6 NSCLC patients, including 4 LUAD and 2 LUSC cases (see Table S1). To obtain CTCs, blood samples were drawn from the 6 patients who had been newly diagnosed with lung cancer and processed using the immunostaining-FISH-based CTC technique (18-21), a CD45-based immunomagnetic system that combines leukocyte common antigen (CD45) immunostaining, and FISH using unprocessed blood samples that was specifically adapted to achieve a capture rate of $>98 \%$ for single CTC and CTC clusters $(18,22)$. Upon capture, the fixed CTCs were stained with antibodies against CD45 to identify contaminating leukocytes (see Figure S1). Upon staining verification, we identified CTCs in the 6 patients; the CTCs from each patient were individually captured and deposited in $10 \mu \mathrm{L}$ of lysis buffer for WGBS $(18,22)$. The WGBS sequencing data comprised $30 \mathrm{G}$ raw data (10x) for the tissue samples and bulk WBCs, and $90 \mathrm{G}$ raw data $(30 \times)$ for the CTC samples. On average, we achieved $47.3 \% \mathrm{CpG}$ 
coverage for CTCs, which is in line with a recent single-cell WGBS study (12). For each individual methylation profile, only CpG sites $\geq 3 \times$ coverage were used for the clustering and functional analyses (see Table S5).

To refine the tumor methylation signature in the matched CTCs, we identified similar methylation CpGs (SMCs) with methylation differences $<10 \%$ and $\mathrm{P}$ values $>0.05$ between CTCs and the matched tumor tissue for each patient and included 450K CpG sites (see Table S6). Thus, we identified the $\mathrm{CT}_{450 \mathrm{~K}}$ SMCs present in both the CTCs and tumor methylation profiles with similar methylation levels that were also included in the $450 \mathrm{~K} \mathrm{CpG}$ sites. Next, we merged the $\mathrm{CT}_{450 \mathrm{~K}} \mathrm{SMCs}$ from the 6 patients and used these $\mathrm{CpG}$ sites as features to cluster matched normal and lung cancer tissues, WBCs, and CTCs. After the refinement of the $\mathrm{CT}_{450 \mathrm{~K}}$ methylation signatures, all 6 pairs of CTCs and matched tumor tissues clustered together, and the Pearson correlation coefficient of CTCs and tumor tissues for each patient was $>0.994$ (see Figure 3A). These results suggest that CTCs inherit most of their methylation signatures from the primary tumors.

However, clustering of the $\mathrm{CT}_{450 \mathrm{~K}}$ methylation analysis did not group the CTCs and tumors together. The high Pearson correlation coefficient of CTCs and WBCs/normal led us to consider the existence of WBCs and normal tissue backgrounds in the CTC methylation pattern. To eliminate WBC backgrounds in the CTC methylation profile, we identified CTC/WBC/Tumor $(\mathrm{CBT})_{450 \mathrm{~K}} \mathrm{DMCs}$ for each patient (see Table S6). Next, we merged the $\mathrm{CBT}_{450 \mathrm{~K}} \mathrm{DMCs}$ of the 6 patients together, and used these merged DMCs to cluster the lung cancer tissues and matched normal tissues/WBCs/CTCs. The Pearson correlation coefficient of the CTCs and WBCs for each patient decreased from a minimum of 0.831 (see Figure 3 B) to a maximum of -0.696 (see Figure 3C). The results showed that almost all 6 pairs of CTCs and matched tumors clustered together, except for the clustering of $2 \mathrm{~T}$ and $2 \mathrm{C}$ due to the ineffective bisulfite conversion of $2 \mathrm{~B}$ (conversion ratio $91.46 \%$ ). Figure $3 C$ shows that the WBC background is an important component of the CTC methylation pattern.

To further eliminate the normal tissue background from the CTC methylation profile, we identified CTC/WBC/ Tumor/Normal $(\mathrm{CBTN})_{450 \mathrm{~K}} \mathrm{DMCs}$ in each patient (see Table S6), and then merged the $\mathrm{CBTN}_{450 \mathrm{~K}}$ DMCs of the 6 patients together and used these merged DMCs to cluster the matched normal and lung cancer tissues, WBCs, and CTCs (see Figure 3D). The poor clustering performance suggested that a normal tissue background is not an effective component of the CTC methylation pattern.

Based on the above methylation profiles, our NSCLC tissue cohort showed that the methylation panel for all LUAD, LUSC, and normal tissues had a diagnostic accuracy of $100 \%$ (see Table S7); after removing the matched WBC background, the diagnostic accuracy of the methylation panel for LUAD and LUSC was also $100 \%$ based on the CTC cohort (see Table S8) (C\&B diff $>0.1$, $\mathrm{P}<0.05)$.

\section{Inberitance of CTCs}

To explore the characteristics of CTCs, we specifically investigated methylation profile distribution according to functional genomic features between CTCs and matched tumor tissues (see Figure 4). We observed that the number of $\mathrm{CpG}$ sites in each CTC/Tumor (CT) similar group was far larger than that in the matched CT difference group (see Figure 4A), which implies that CTCs have a large proportion of methylation signatures inherited from the primary tumor. In addition, 5-methylcytosine (5-mC) was most common in TFBSs and intronic and intergenic regions, accounting for $42.8 \%, 47.3 \%$, and $46.0 \%$ of all CpG sites in tumors, and $47.4 \%, 47.6 \%$, and $42.8 \%$ of all CpG sites in CTCs, respectively (see Figure 4A). 5-mC was also commonly found in enhancers, superenhancers, promoters, and $\mathrm{CpG}$ shores, accounting for $5.6 \%$, $24.8 \%, 5.5 \%$, and $6.6 \%$ of all CpG sites in tumors, and $6.1 \%, 28.6 \%, 9.1 \%$, and $8.0 \%$ of all CpG sites in CTCs, respectively (see Figure $4 A$ ).

For regulatory elements, the loss of DNA methylation at TFBSs can designate active transcription factor networks or networks primed for activation at later stages [e.g., during processes such as the derivation of induced pluripotent stem cells from differentiated cells (23) or cancer progression (9)]. We then analyzed SMCs at TFBSs using i-Cistarget (17), and used the clusterProfile R package to analyze the KEGG pathways of global CTC hypomethylated TFBSs coexisting in CTCs and matched tumor tissues with methylation differences $<10 \%$ and $\mathrm{P}$ values $>0.05$ (see Figure $5 A$ ). Our DNA methylation analysis revealed the mitogen-activated protein kinase signaling pathway and pathways regulating the pluripotency of stem cells, epithelial growth factor receptor (EGFR) tyrosine kinase inhibitor resistance, and the cell cycle. These pathways coexisted in both CTCs and matched tumor tissues, suggesting that CTC methylation originates from primary tumor tissues and is inherited as the cells move from the primary tumor tissues to the peripheral 

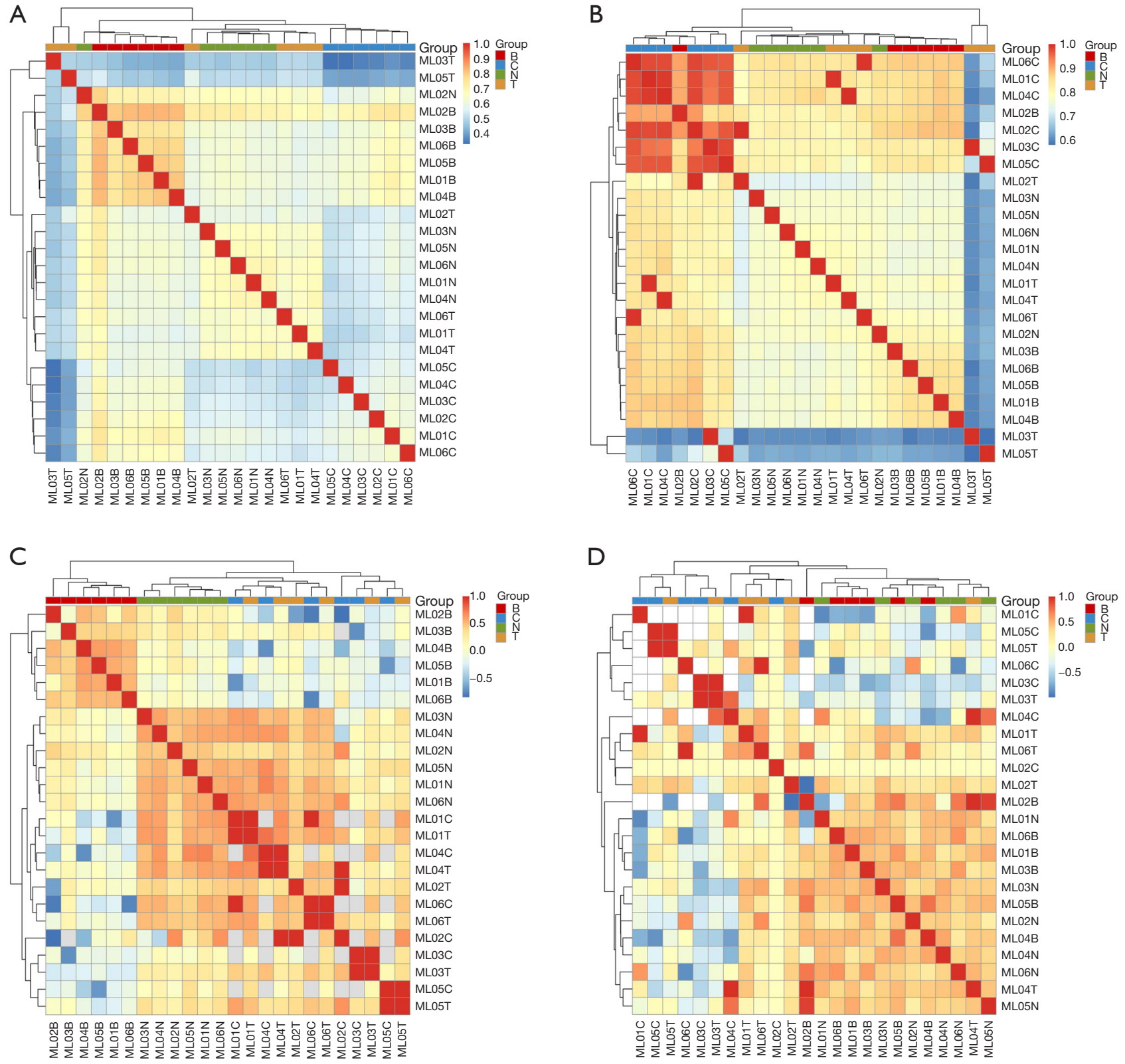

Figure 3 Unsupervised hierarchical clustering associated with the methylation profile (A) included in the 450K methylation array (according to the color scale shown) in cancer tissue (T), normal tissue (N), WBC bulk (B), and CTC (C) data for 6 patients. (B) Included in the CTC/ Tumor (CT) similar methylation markers selected for use in the 24 samples from the 6 patients. (C) Included in the CBT methylation markers selected for use in the 24 samples from the 6 patients. (D) Included in the CTC/WBC/Tumor/Normal (CBNT) methylation markers selected for use in the 24 samples from the 6 patients. The color scale shows the Pearson correlation coefficients. CTC, circulating tumor cell.

blood (see Figure 5A). We also found that binding sites for stemness-associated transcription factors are specifically hypomethylated in SMCs in both CTCs and matched tumor tissues, including binding sites for NANOG and
SOX2, which in previous reports were associated with CTC clusters compared to single CTCs (24).

To explore other subtle changes in DNA methylation occurring specifically within promoters, gene bodies, and 

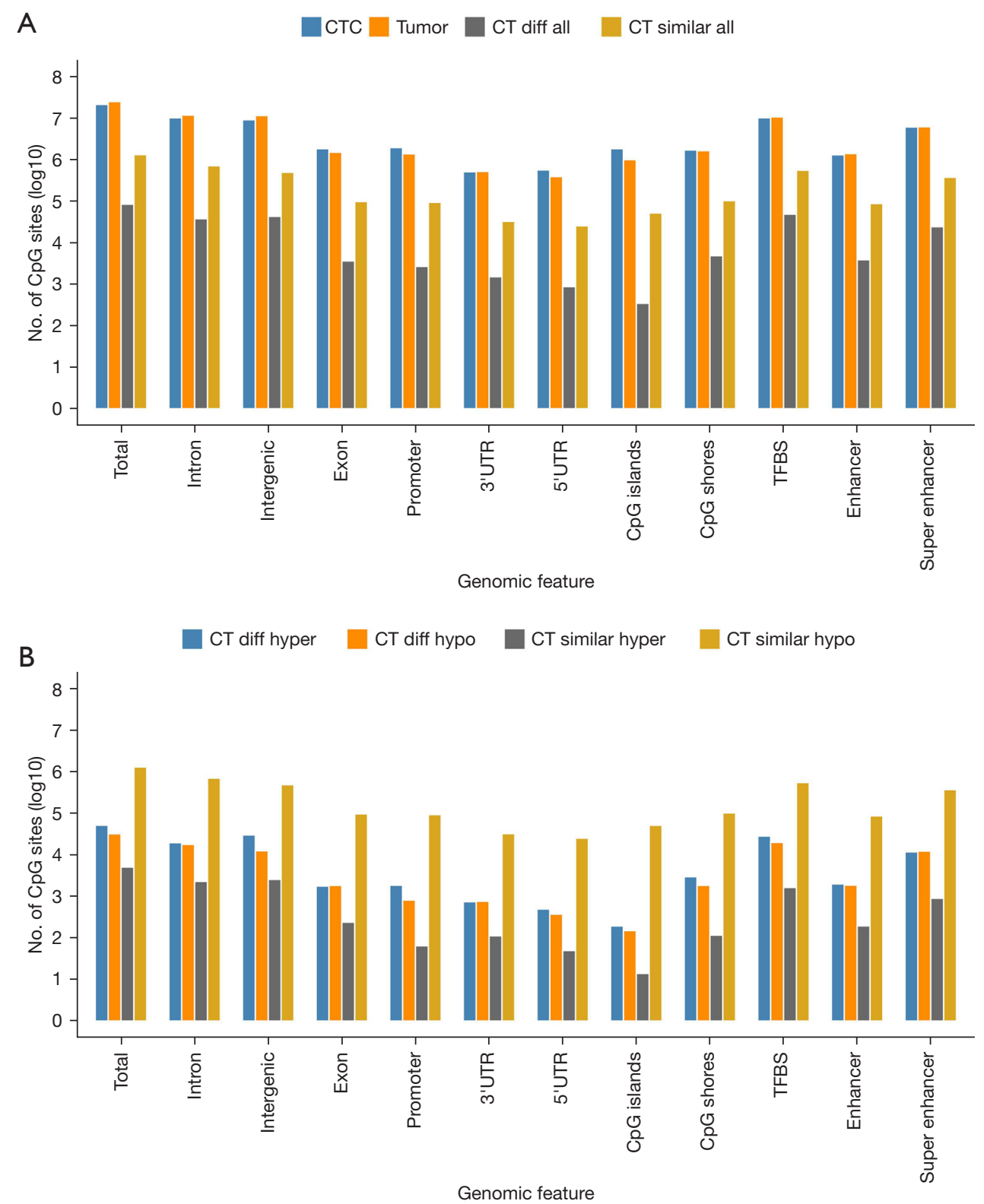

Figure $4 \mathrm{CpG}$ sites with known genomic features in 6 patients. $\mathrm{CpG}$ sites with no less than $3 \times$ coverage were counted, as shown in this figure. Similar CpG sites (CT similar) corresponding to genomic features with a normal $\mathrm{P}$ value $>0.05$ and a methylation difference $<10 \%$ were counted between the CTCs and matched tumor tissues. Differential CpG sites (CT diff) corresponding to genomic features with a normal $\mathrm{P}$ value $<0.05$ and an absolute methylation difference $>0.1$ were counted in the CTCs compared to matched tumor tissues. The DMCs that appeared in 2 of the 6 patients are illustrated in this figure. CTC, circulating tumor cell.

superenhancer regions, we carried out a hypergeometricbased gene set enrichment analysis of genomic features. Consistently, this analysis revealed hypomethylation and cell cycle progression (see Figure S2), as previously observed for cancer specimens with stem-like and proliferative features.

\section{Evolution of CTCs}

The CTCs showed many DMCs differed to those of the primary tumor (see Figure 4B). To identify whether the characteristic-related transcription factor networks were also transcriptionally active in CTCs compared to matched 

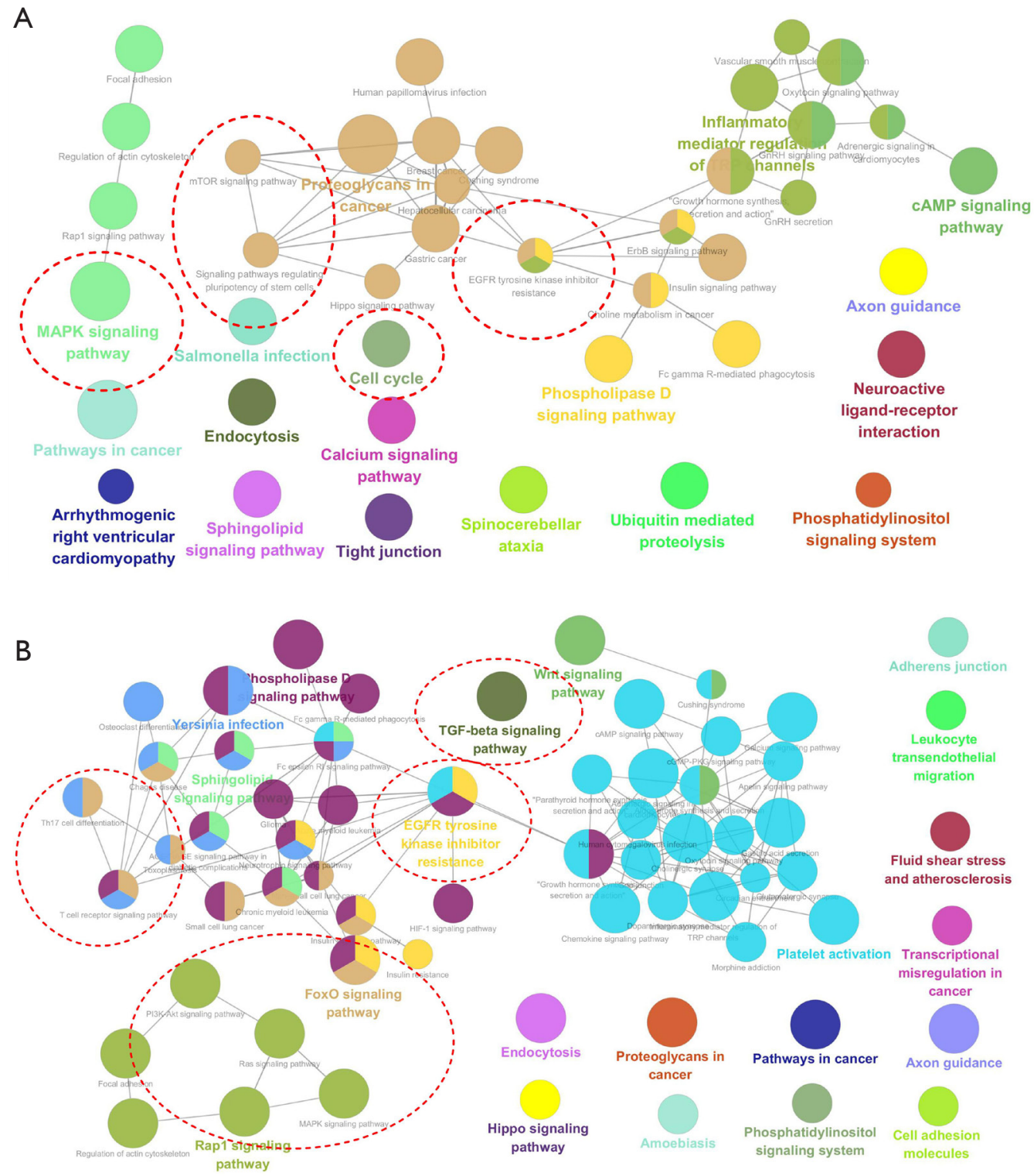

Figure 5 Pathway enrichment analysis of TFBSs on a genome-wide scale identified using i-cis Target in hypomethylated regions of CTsimilar (A) and CT-differential (B). CT-similar displayed a $<10 \%$ methylation difference (P value $>0.05)$ in CTCs compared to matched tumor tissues among the 6 patients. CT-differential displayed a $>0.1$ methylation difference $(\mathrm{P}$ value $<0.05)$ in CTCs compared to matched tumor tissues among the 6 patients. Gene sets with an adjusted $\mathrm{P}$ value $<0.01$ were considered significant. TFBS, transcription factor binding site; CTC, circulating tumor cell.

tumors, we performed a single-cell resolution methylation sequencing analysis of CTCs and matched tumors isolated from the 6 NSCLC patients (see Figure 5). We used the cluster Profile R package to analyze the KEGG pathways of global hypomethylated TFBSs in CTCs rather than matched tumor tissues with an absolute methylation difference $>0.1$ and a $\mathrm{P}$ value $<0.05$ (see Figure $5 B$ ). The
KEGG analysis of TFBS DMCs in patient CTCs compared to matched tumors revealed the enrichment of genes related to the $\mathrm{T}$ cell receptor signaling pathway, Th17 cell differentiation, transforming growth factor (TGF)beta signaling pathway, EGFR tyrosine kinase inhibitor resistance, and canonical pathways (RAS/MAPK/PI3K/ AKT) (see Figure 5B). 
In relation to the other subtle changes in DNA methylation occurring specifically in promoters, gene bodies, and superenhancer regions, the KEGG analysis revealed the enrichment of gene groups related to the TGF-beta signaling pathway, Th17 cell differentiation, $\mathrm{T}$ cell receptor signaling pathway, and EGFR tyrosine kinase inhibitor resistance (see Figure S3).

These results show that in the processes of dissemination from the primary tumor tissue to the peripheral blood, CTCs gradually develop their own unique methylation signatures with some unique characteristics that differ to those of the primary tumor.

\section{Discussion}

Our study shows that CTC methylation signatures can be used as an alternative non-invasive approach to biopsy for the pathological classification of NSCLC patients. We found that these methylation signatures can identify LUAD and LUSC with extremely high accuracy. The histologic definition of NSCLC is of huge importance to drive molecular testing and optimize treatment selection, which is a non-invasive method for histologic diagnosis. Our results raise the possibility that the detection of CTC methylation in peripheral blood may be expanded to aid in the diagnosis of a much larger number of tumor types. In addition, we uncovered 2 CTC methylation patterns of inheritance and evolution during the CTC migration process. These features of CTCs provide new insights into the mechanism of NSCLC metastasis.

In practice, the amount of genomic DNA in CTCs per patient is typically limited to a range of 10 s of picograms. Moreover, the amplification of damaged DNA beginning with a fixed CTC cell is even more difficult than the amplification of integrated genomic DNA beginning with a live cell. In our study, we collected all the CTCs from each patient into 1 tube. We then amplified and achieved an average of $47.3 \% \mathrm{CpG}$ coverage for CTCs, which is similar to the $50 \% \mathrm{CpG}$ site coverage in a single cell reported by a recent study (12).

Our study suggests that CTCs share several properties common to immune escape and in mesenchymal-shifted cells compared to matched tumors. For example, the T-cell receptor signaling pathway and Th17 cell differentiation contributes to tumor immune escape (25-33). The TGFbeta pathway promotes epithelial-mesenchymal transition (EMT) in tumor cells, which plays an important role in mediating tumor invasion and metastasis $(34,35)$. EGFR tyrosine kinase inhibitor resistance suggests a form of acquired drug resistance, which is associated with the tumor cell EMT phase (36-38). The canonical RAS/ MAPK/PI3K/AKT signaling pathway is involved in EMT progression (39). Previous reports have demonstrated that the enhancement of mesenchymal-like features epigenetically reprograms epithelial cancer cells to adapt well to new microenvironments, and thus may contribute to distant metastasis (40). Several reports have focused on the relationship between EMT and immune escape $(39,41-44)$, especially in NSCLC. However, previous studies have only focused on a few genes or proteins in CTCs associated with immune escape, such as the upregulation of CD47 expression as a potential escape mechanism in colorectal cancer based on quantitative polymerase chain reaction (45) or the downregulation of ULBP1 protein expression as a potential CTC evasion mechanism from natural killer cells (46). Our study uncovered a CTC immune escape mechanism through CTC methylation signatures on a genome-wide scale, and we propose that the EMT status of CTCs and T-cell receptor signaling ultimately leads to tumor immune escape and invasion.

The methylation profiling of circulating tumor DNA has been investigated in cancer diagnostics and in the assessment of therapeutic outcomes (47-52), but to date, few methylation profiles of CTCs have been studied and derived a DNA methylation signature in CTCS of patients with lung cancer. We used CTC methylation profiles to identify LUAD and LUSC with extremely high accuracy in 6 NSCLC patients. In our study, CTCs, as 1 of the 3 liquid biopsy biomarker types, showed strong potential in terms of methylation origin and classification. Compared to circulating tumor DNA and exosomes, CTCs carry a complete genome, which provides an incomparable advantage. Our study demonstrated that CTC traceability only requires deducing the matched WBCs. Conversely, circulating tumor DNA traceability needs a large training set and complex algorithm due to its rarity in the blood.

As an auxiliary diagnosis tool for benign and malignant lesions, CTC techniques should be strengthened. However, the capture of CTC remains a huge challenge for the widely available in the cancer diagnosis and other technologies, such as microfluidic technology, may be used to count and capture CTCs with both high sensitivity and specificity and low damage to the cells. Further, more analytical methods and models should be explored to improve coverage or change the analysis units from methylated cytosines to methylated regions $(24,53)$. 


\section{Conclusions}

In summary, our study provides insights into the potential of CTCs to replace invasive biopsy for the pathological classification of NSCLC patients. Further, we also found that CTC primary tumor inheritance and CTC evolution affect metastasis and immune escape.

\section{Acknowledgments}

The authors appreciate the academic support from the AME Lung Cancer Collaborative Group.

Funding: This work was supported by the National Natural Science Foundation of China (81972168), and the National Key Research and Development Program of China (2016YFA0502202, HW).

\section{Footnote}

Reporting Checklist: The authors have completed the MDAR reporting checklist. Available at https://tlcr.amegroups.com/ article/view/10.21037/tlcr-22-50/rc

Data Sharing Statement: Available at https://tlcr.amegroups. com/article/view/10.21037/tlcr-22-50/dss

Conflicts of Interest: All authors have completed the ICMJE uniform disclosure form (available at https://tlcr.amegroups. com/article/view/10.21037/tlcr-22-50/coif). CYC, JL, and $\mathrm{YL}$ are from Shanghai Zhiyi Biomedical Technology Company, WF is from Jilin Province JinKangAn Pharmaceutical Company. The other authors have no conflicts of interest to declare.

Ethical Statement: The authors are accountable for all aspects of the work in ensuring that questions related to the accuracy or integrity of any part of the work are appropriately investigated and resolved. Written informed consent was obtained from the patients, and ethical approval was obtained from the Zhongshan Hospital Research Ethics Committee (No. Y2019-187). All procedures performed in this study involving human participants were in accordance with the Declaration of Helsinki (as revised in 2013).

Open Access Statement: This is an Open Access article distributed in accordance with the Creative Commons Attribution-NonCommercial-NoDerivs 4.0 International License (CC BY-NC-ND 4.0), which permits the noncommercial replication and distribution of the article with the strict proviso that no changes or edits are made and the original work is properly cited (including links to both the formal publication through the relevant DOI and the license). See: https://creativecommons.org/licenses/by-nc-nd/4.0/.

\section{References}

1. Sung H, Ferlay J, Siegel RL, et al. Global Cancer Statistics 2020: GLOBOCAN Estimates of Incidence and Mortality Worldwide for 36 Cancers in 185 Countries. CA Cancer J Clin 2021;71:209-49.

2. Reck M, Rabe KF. Precision Diagnosis and Treatment for Advanced Non-Small-Cell Lung Cancer. N Engl J Med 2017;377:849-61.

3. Zhang Z, Xiao Y, Zhao J, et al. Relationship between circulating tumour cell count and prognosis following chemotherapy in patients with advanced non-small-cell lung cancer. Respirology 2016;21:519-25.

4. Matthew EM, Zhou L, Yang Z, et al. A multiplexed marker-based algorithm for diagnosis of carcinoma of unknown primary using circulating tumor cells. Oncotarget 2016;7:3662-76.

5. Vidal E, Sayols S, Moran S, et al. A DNA methylation map of human cancer at single base-pair resolution. Oncogene 2017;36:5648-57.

6. Ehrlich M. DNA methylation in cancer: too much, but also too little. Oncogene 2002;21:5400-13.

7. Ehrlich M. DNA hypomethylation in cancer cells. Epigenomics 2009;1:239-59.

8. Farlik M, Halbritter F, Müller F, et al. DNA Methylation Dynamics of Human Hematopoietic Stem Cell Differentiation. Cell Stem Cell 2016;19:808-22.

9. Feinberg AP, Vogelstein B. Hypomethylation distinguishes genes of some human cancers from their normal counterparts. Nature 1983;301:89-92.

10. Fernandez AF, Assenov Y, Martin-Subero JI, et al. A DNA methylation fingerprint of 1628 human samples. Genome Res 2012;22:407-19.

11. Moran S, Martínez-Cardús A, Sayols S, et al. Epigenetic profiling to classify cancer of unknown primary: a multicentre, retrospective analysis. Lancet Oncol 2016;17:1386-95.

12. Clark SJ, Smallwood SA, Lee HJ, et al. Genome-wide base-resolution mapping of DNA methylation in single cells using single-cell bisulfite sequencing (scBS-seq). Nat Protoc 2017;12:534-47.

13. Krueger F, Andrews SR. Bismark: a flexible aligner and methylation caller for Bisulfite-Seq applications. 
Bioinformatics 2011;27:1571-2.

14. Langmead B, Salzberg SL. Fast gapped-read alignment with Bowtie 2. Nat Methods 2012;9:357-9.

15. Akalin A, Kormaksson M, Li S, et al. methylKit: a comprehensive $\mathrm{R}$ package for the analysis of genome-wide DNA methylation profiles. Genome Biol 2012;13:R87.

16. Wang HQ, Tuominen LK, Tsai CJ. SLIM: a sliding linear model for estimating the proportion of true null hypotheses in datasets with dependence structures. Bioinformatics 2011;27:225-31.

17. Herrmann C, Van de Sande B, Potier D, et al. i-cisTarget: an integrative genomics method for the prediction of regulatory features and cis-regulatory modules. Nucleic Acids Res 2012;40:e114.

18. Wan JF, Li XQ, Zhang J, et al. Aneuploidy of chromosome 8 and mutation of circulating tumor cells predict pathologic complete response in the treatment of locally advanced rectal cancer. Oncol Lett 2018;16:1863-8.

19. Lin PP, Gires O, Wang DD, et al. Comprehensive in situ co-detection of aneuploid circulating endothelial and tumor cells. Sci Rep 2017;7:9789.

20. Lin PP. Erratum to: Integrated EpCAM-independent subtraction enrichment and iFISH strategies to detect and classify disseminated and circulating tumors cells. Clin Transl Med 2016;5:6.

21. Lin PP. Integrated EpCAM-independent subtraction enrichment and iFISH strategies to detect and classify disseminated and circulating tumors cells. Clin Transl Med 2015;4:38.

22. Chen C, Li J, Wan J, et al. A low cost and input tailing method of quality control on multiple annealing, and looping-based amplification cycles-based whole-genome amplification products. J Clin Lab Anal 2019;33:e22697.

23. Lee DS, Shin JY, Tonge PD, et al. An epigenomic roadmap to induced pluripotency reveals DNA methylation as a reprogramming modulator. Nat Commun 2014;5:5619.

24. Gkountela S, Castro-Giner F, Szczerba BM, et al. Circulating Tumor Cell Clustering Shapes DNA Methylation to Enable Metastasis Seeding. Cell 2019;176:98-112.e14.

25. Han Y, Ye A, Bi L, et al. Th17 cells and interleukin-17 increase with poor prognosis in patients with acute myeloid leukemia. Cancer Sci 2014;105:933-42.

26. Kim JM, Chen DS. Immune escape to PD-L1/PD-1 blockade: seven steps to success (or failure). Ann Oncol 2016;27:1492-504.

27. Grywalska E, Pasiarski M, Góźdź S, et al. Immunecheckpoint inhibitors for combating T-cell dysfunction in cancer. Onco Targets Ther 2018;11:6505-24.

28. He X, Xu C. Immune checkpoint signaling and cancer immunotherapy. Cell Res 2020;30:660-9.

29. Price DA, West SM, Betts MR, et al. T cell receptor recognition motifs govern immune escape patterns in acute SIV infection. Immunity 2004;21:793-803.

30. Qin A, Coffey DG, Warren EH, et al. Mechanisms of immune evasion and current status of checkpoint inhibitors in non-small cell lung cancer. Cancer Med 2016;5:2567-78.

31. Reuben A, Zhang J, Chiou SH, et al. Comprehensive T cell repertoire characterization of non-small cell lung cancer. Nat Commun 2020;11:603.

32. Spranger S. Mechanisms of tumor escape in the context of the T-cell-inflamed and the non-T-cell-inflamed tumor microenvironment. Int Immunol 2016;28:383-91.

33. Tian Y, Zhai X, Han A, et al. Potential immune escape mechanisms underlying the distinct clinical outcome of immune checkpoint blockades in small cell lung cancer. J Hematol Oncol 2019;12:67.

34. Colak S, Ten Dijke P. Targeting TGF-beta signaling in cancer. Trends Cancer 2017;3:56-71.

35. Drabsch Y, Ten Dijke P. TGF-beta signalling and its role in cancer progression and metastasis. Cancer Metastasis Rev 2012;31:553-68.

36. Gainor JF, Dardaei L, Yoda S, et al. Molecular Mechanisms of Resistance to First- and Second-Generation ALK Inhibitors in ALK-Rearranged Lung Cancer. Cancer Discov 2016;6:1118-33.

37. Liu X, Li J, Cadilha BL, et al. Epithelial-type systemic breast carcinoma cells with a restricted mesenchymal transition are a major source of metastasis. Sci Adv 2019;5:eaav4275.

38. Fischer KR, Durrans A, Lee S, et al. Epithelial-tomesenchymal transition is not required for lung metastasis but contributes to chemoresistance. Nature 2015;527:472-6.

39. Jiang Y, Zhan H. Communication between EMT and PDL1 signaling: New insights into tumor immune evasion. Cancer Lett 2020;468:72-81.

40. Mitra A, Mishra L, Li S. EMT, CTCs and CSCs in tumor relapse and drug-resistance. Oncotarget 2015;6:10697-711.

41. Mak MP, Tong P, Diao L, et al. A Patient-Derived, PanCancer EMT Signature Identifies Global Molecular Alterations and Immune Target Enrichment Following Epithelial-to-Mesenchymal Transition. Clin Cancer Res 2016;22:609-20.

42. Lou Y, Diao L, Cuentas ER, et al. Epithelial-Mesenchymal 
Transition Is Associated with a Distinct Tumor

Microenvironment Including Elevation of Inflammatory

Signals and Multiple Immune Checkpoints in Lung

Adenocarcinoma. Clin Cancer Res 2016;22:3630-42.

43. Kim S, Koh J, Kim MY, et al. PD-L1 expression is associated with epithelial-to-mesenchymal transition in adenocarcinoma of the lung. Hum Pathol 2016;58:7-14.

44. Tiwari N, Gheldof A, Tatari M, et al. EMT as the ultimate survival mechanism of cancer cells. Semin Cancer Biol 2012;22:194-207.

45. Steinert G, Schölch S, Niemietz T, et al. Immune escape and survival mechanisms in circulating tumor cells of colorectal cancer. Cancer Res 2014;74:1694-704.

46. Hu B, Tian X, Li Y, et al. Epithelial-mesenchymal transition may be involved in the immune evasion of circulating gastric tumor cells via downregulation of ULBP1. Cancer Med 2020;9:2686-97.

47. Taylor WC. Comment on 'Sensitive and specific multicancer detection and localization using methylation signatures in cell-free DNA' by M. C. Liu et al. Ann Oncol 2020;31:1266-7.

Cite this article as: Jiang JH, Gao J, Chen CY, Ao YQ, Li J, Lu Y, Fang W, Wang HK, de Castro DG, Santarpia M, Hashimoto M, Yuan YF, Ding JY. Circulating tumor cell methylation profiles reveal the classification and evolution of non-small cell lung cancer. Transl Lung Cancer Res 2022;11(2):224-237. doi: 10.21037/tlcr-22-50
48. Liu L, Toung JM, Jassowicz AF, et al. Targeted methylation sequencing of plasma cell-free DNA for cancer detection and classification. Ann Oncol 2018;29:1445-53.

49. Jiang P, Sun K, Tong YK, et al. Preferred end coordinates and somatic variants as signatures of circulating tumor DNA associated with hepatocellular carcinoma. Proc Natl Acad Sci U S A 2018;115:E10925-33.

50. Xu RH, Wei W, Krawczyk M, et al. Circulating tumour DNA methylation markers for diagnosis and prognosis of hepatocellular carcinoma. Nat Mater 2017;16:1155-61.

51. Guo S, Diep D, Plongthongkum N, et al. Identification of methylation haplotype blocks aids in deconvolution of heterogeneous tissue samples and tumor tissue-of-origin mapping from plasma DNA. Nat Genet 2017;49:635-42.

52. Lee EJ, Luo J, Wilson JM, et al. Analyzing the cancer methylome through targeted bisulfite sequencing. Cancer Lett 2013;340:171-8.

53. Hao X, Luo H, Krawczyk M, et al. DNA methylation markers for diagnosis and prognosis of common cancers. Proc Natl Acad Sci U S A 2017;114:7414-9. 
Supplementary

Table S1 Information for the patients enrolled in this study

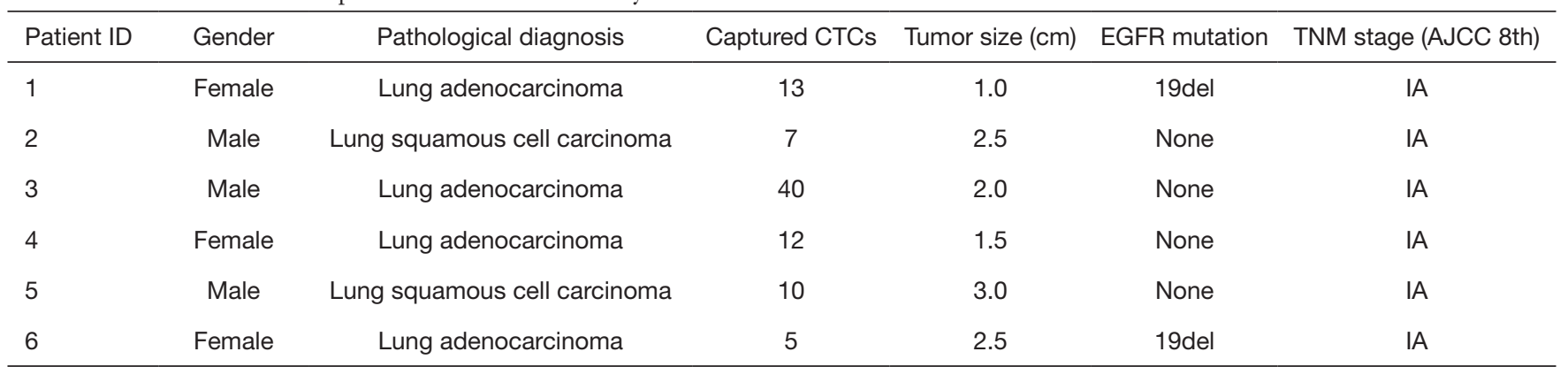

CTC, circulating tumor cell; EGFR, epidermal growth factor receptor; AJCC, American Joint Committee on Cancer; TNM, tumor node metastasis.

Table S2 Confusion table of training cohort

\begin{tabular}{lcccc}
\hline Training & LUAD & LUSC & Normal lung & Total \\
\hline LUAD & 310 & 7 & 0 & 317 \\
LUSC & 5 & 225 & 0 & 230 \\
Normal lung & 3 & 3 & 60 & 66 \\
Total & 318 & 235 & 60 & 613 \\
Correct & 310 & 225 & 60 & 595 \\
Correct $(\%)$ & 97.48428 & 95.74468 & 100 & 97.06362 \\
\hline
\end{tabular}

LUAD, lung adenocarcinoma; LUSC, lung squamous cell carcinoma. 
Table S3 Confusion table of validation cohort 1

\begin{tabular}{lcccc}
\hline Validation 1 & LUAD & LUSC & Normal lung & Total \\
\hline LUAD & 151 & 5 & 0 & 156 \\
LUSC & 2 & 127 & 0 & 129 \\
Normal lung & 1 & 2 & 14 & 302 \\
Total & 154 & 134 & 14 & 292 \\
Correct & 151 & 127 & 14 & 96.68874 \\
Correct (\%) & 98.05195 & 94.77612 & 100 \\
\hline
\end{tabular}

LUAD, lung adenocarcinoma; LUSC, lung squamous cell carcinoma.

Table S4 Confusion table of validation cohort 2

\begin{tabular}{lcccc}
\hline Validation 2 & LUAD & LUSC & Normal lung & Total \\
\hline LUAD & 25 & 1 & 1 & 27 \\
LUSC & 2 & 7 & 73 & 9 \\
Normal lung & 2 & 0 & 74 & 111 \\
Total & 29 & 8 & 73 & 105 \\
Correct & 25 & 7 & 98.64865 & 94.59459 \\
Correct (\%) & 86.2069 & 87.5 & & 75 \\
\hline
\end{tabular}

LUAD, lung adenocarcinoma; LUSC, lung squamous cell carcinoma. 


\section{A \\ CTC \\ (DAPI+/CD45-I \\ CEP8 $\geq 3$ )}

B

\section{WBC \\ (DAPI+/CD45+/ \\ CEP8=2)}
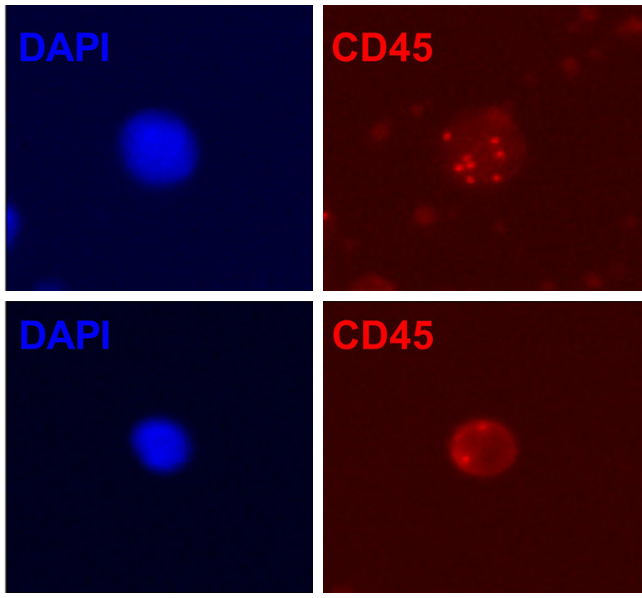
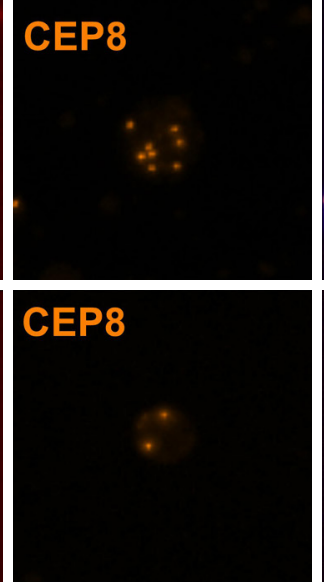

Merged

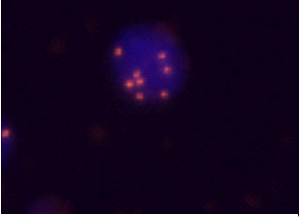

Merged

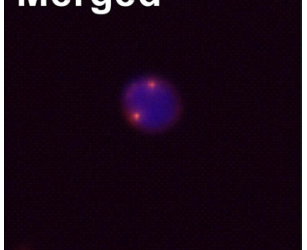

Figure S1 In situ phenotypic and karyotypic characterization of aneuploid CTCs. CTCs are DAPI+ (blue)/CD45-/FISH+ (aneuploid chromosome 8 , orange) $\geq 3$. WBCs are DAPI+ (blue)/CD45+/FISH- (aneuploid chromosome 8 , orange) $=2$. The picture was magnified with $40 \times$ under the fluorescence microscope. 
Table S5 Summary of the basic sequencing parameters, including the sequencing depth, for all 6 patients

\begin{tabular}{|c|c|c|c|c|c|c|}
\hline Case No. & Raw_bases & Conversion rate (\%) & Map-ability (\%) & Duplication rate (\%) & Sequence depth & 1xCpG coverage \\
\hline 01B & 34023049106 & 99.51 & 88.62 & 16.50 & 7.001361 & 95.608 \\
\hline 02B & 31388659920 & 91.46 & 29.14 & 52.24 & 1.156164 & 44.667 \\
\hline 03B & 33872628040 & 99.77 & 87.78 & 18.54 & 6.717153 & 94.709 \\
\hline 04B & 31534218786 & 99.73 & 88.77 & 16.83 & 6.30206 & 93.076 \\
\hline 06B & 33407950002 & 99.72 & 88.71 & 22.55 & 6.349538 & 93.927 \\
\hline $01 \mathrm{~N}$ & 40379812376 & 99.68 & 87.23 & 21.45 & 7.645562 & 93.645 \\
\hline $02 \mathrm{~N}$ & 1436336462 & 98.76 & 81.85 & 32.54 & 0.202227 & 14.748 \\
\hline 03N & 34455483812 & 99.69 & 85.31 & 17.22 & 6.717548 & 92.865 \\
\hline $01 T$ & 34563878558 & 99.65 & 87.21 & 19.57 & 6.687883 & 92.707 \\
\hline 02T & 31370116818 & 99.51 & 76.32 & 15.80 & 5.738217 & 92.61 \\
\hline ОЗТ & 34458690146 & 99.72 & 80.61 & 18.77 & 6.189226 & 90.995 \\
\hline 04T & 36849359292 & 99.61 & 88.02 & 19.81 & 7.190256 & 92.878 \\
\hline 05T & 34271216700 & 99.71 & 86.99 & 22.66 & 6.35076 & 91.395 \\
\hline 06T & 38044759550 & 99.68 & 86.86 & 19.38 & 7.373278 & 93.425 \\
\hline $01 \mathrm{C}$ & 90723626720 & 98.99 & 62.06 & 66.06 & 3.856598 & 77.455 \\
\hline
\end{tabular}


Table S6 Parameter conditions for the clustering of cancer tissues, normal tissues, WBCs and CTCs

\begin{tabular}{lccc}
\hline Optimized Condition & CT-similar & CB-diff & CN-diff \\
\hline Raw $_{450 \mathrm{~K}}$ & - & - & - \\
$\mathrm{CT}_{450 \mathrm{~K}}$ & $\mathrm{P}>0.05 ;$ diff $<10 \%$ & - & - \\
$\mathrm{CBT}_{450 \mathrm{~K}}$ & $\mathrm{P}>0.05 ;$ diff $<10 \%$ & $\mathrm{P}<0.05 ;$ diff $>0.15$ & - \\
$\mathrm{CBNT}_{450 \mathrm{~K}}$ & $\mathrm{P}>0.05 ;$ diff $<10 \%$ & $\mathrm{P}<0.05 ;$ diff $>0.15$ & $\mathrm{P}<0.05 ;$ diff $>0.15$ \\
\hline
\end{tabular}

diff, difference.

Table S7 Confusion table of our NSCLC tissue cohort

\begin{tabular}{lcccc}
\hline Validation & LUAD & LUSC & Normal Lung & Total \\
\hline LUAD & 4 & 0 & 0 & \\
LUSC & 0 & 2 & 6 & 12 \\
Normal lung & 0 & 0 & 6 & 12 \\
Total & 4 & 2 & 6 & 100 \\
Correct & 4 & 2 & 100 \\
Correct $(\%)$ & 100 & 100 & 100 \\
\hline
\end{tabular}

NSCLC, non-small cell lung cancer; LUAD, lung adenocarcinoma; LUSC, lung squamous cell carcinoma.

Table S8 Confusion table of the CTC validation cohort, including both $\mathrm{C} \& \mathrm{~B}$ diff $(\mathrm{C} \& \mathrm{~B}$ diff $>0.1, \mathrm{P}<0.05)$ and 5,426 methylation markers

\begin{tabular}{lccc}
\hline Validation & LUAD & LUSC & Total \\
\hline LUAD & 4 & 0 & 2 \\
LUSC & 0 & 0 & 12 \\
Normal lung & 0 & 2 & 12 \\
Total & 4 & 2 & 100 \\
Correct & 4 & 100 & 100 \\
Correct $(\%)$ & 100 & & 12 \\
\hline
\end{tabular}

CTC, circulating tumor cell; diff, difference; LUAD, lung adenocarcinoma; LUSC, lung squamous cell carcinoma. 
A

Hypermethylated

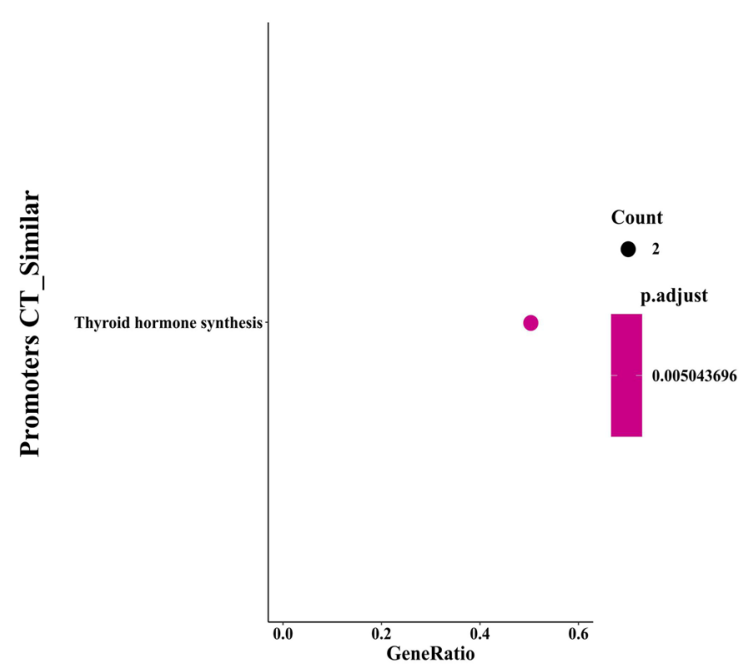

B

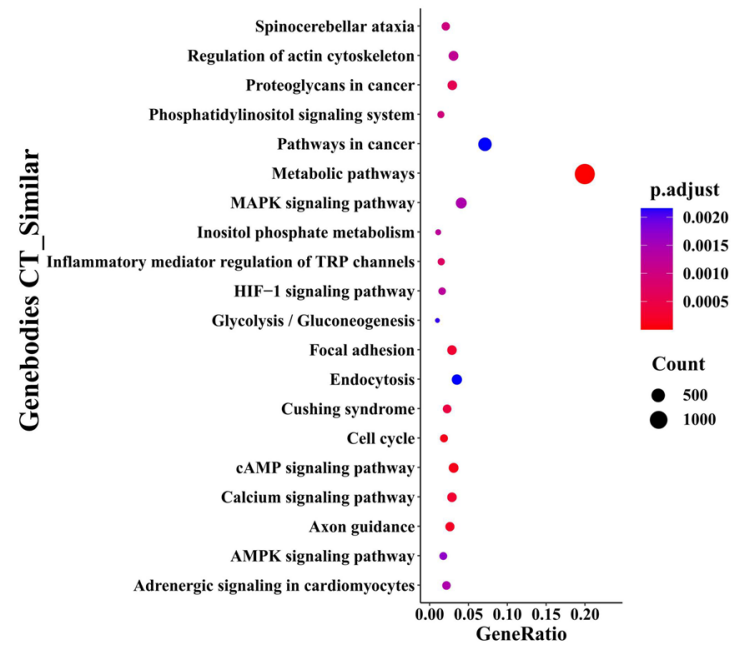

Hypomethylated

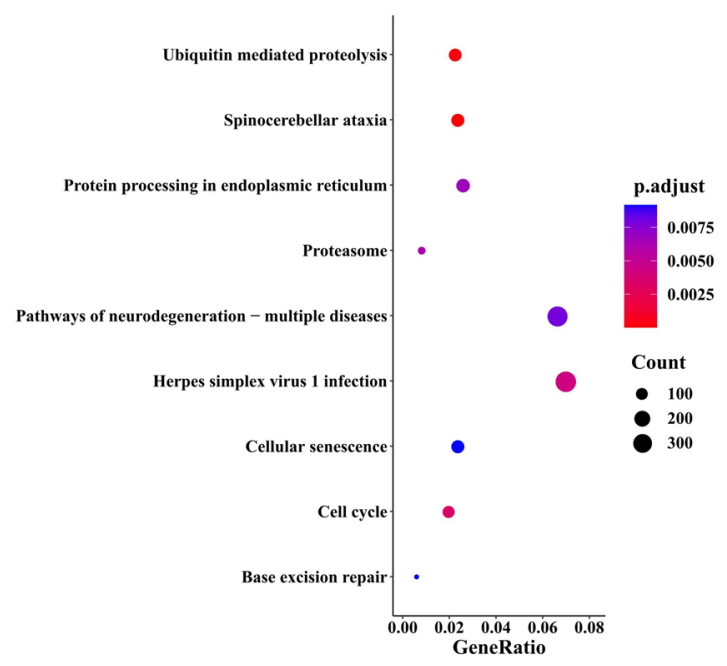

C

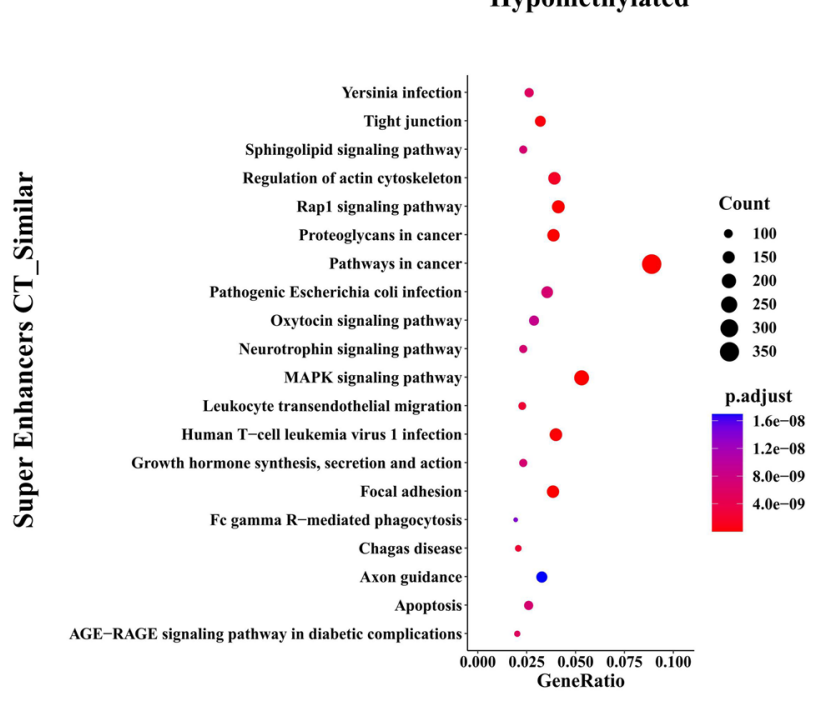

Figure S2 Pathway enrichment analysis of similarly regulated genes between CTCs and matched tumor tissues. The KEGG pathway enrichment of promoter (A) gene bodies (B) and superenhancers (C) displaying a $<10 \%$ methylation difference (P value $>0.05$ ) between CTCs and matched tumor tissues. Gene sets with adjusted $\mathrm{P}$ value $<0.01$ are shown for promoters (A). For hypogene bodies (B) and hyposuperenhancers (C), the top 20 significant gene sets with an adjusted $\mathrm{P}$ value $<0.01$ are shown. For hypergene bodies (B) and hypersuperenhancers (C), no gene had an adjusted $\mathrm{P}$ value $<0.01$. 


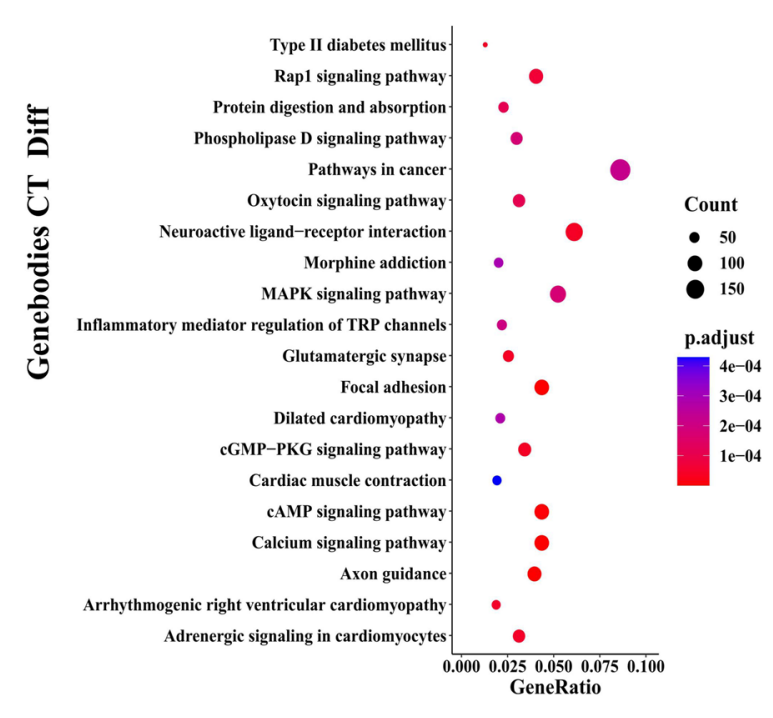

B

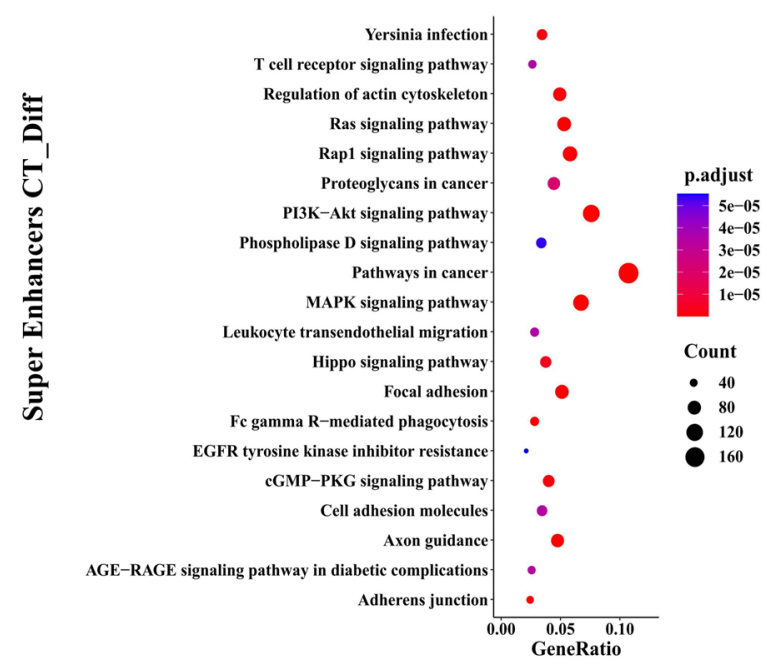

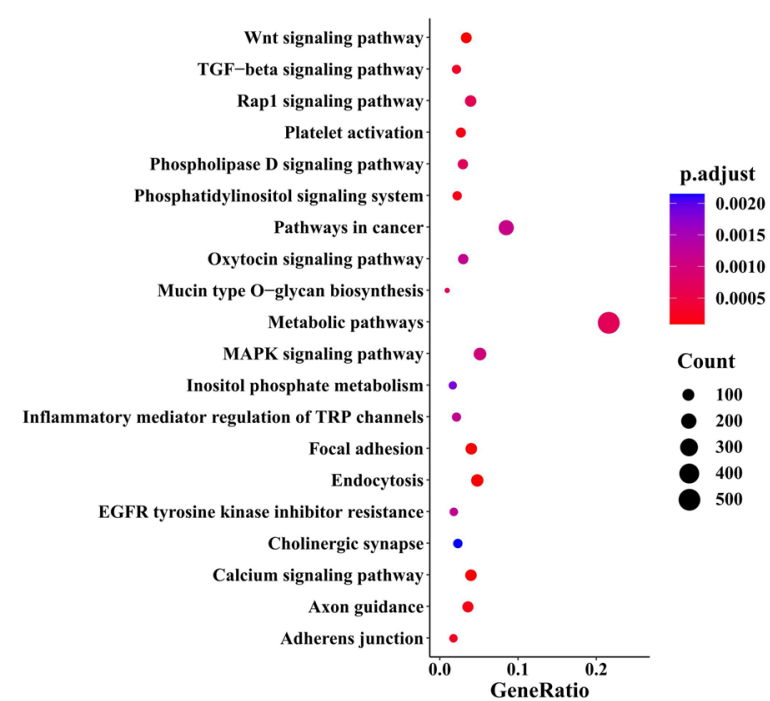

Hypomethylated

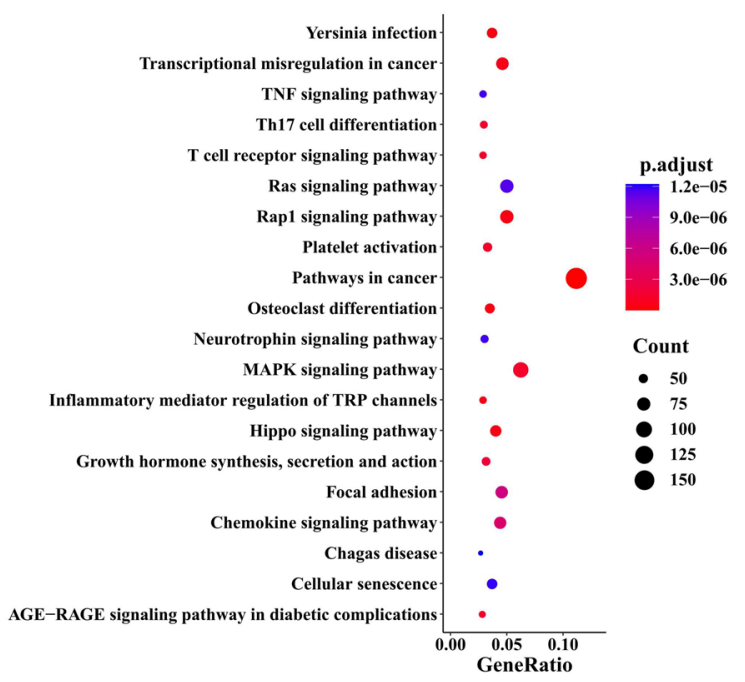

Figure S3 Pathway enrichment analysis for differentially regulated genes from CTCs and matched tumor tissues. The KEGG pathway enrichment of gene bodies (A) and superenhancers (B) displaying $>0.1$ absolute methylation difference (P value $<0.05)$ in CTCs compared to matched tumor tissues among 6 patients. For gene bodies (A) and superenhancers (B), the top 20 significant gene sets with an adjusted $\mathrm{P}$ value $<0.01$ are shown. For promoters (A), no gene had an adjusted $\mathrm{P}$ value $<0.01$. 